\title{
Design of Discrete Wavelet by Using Transient Model for Exact Measurement of Manufacturing Faults of Tapered Roller Bearings
}

\author{
Krisztián Deák ${ }^{1 *}$ \\ ' Department of Mechanical Engineering, Faculty of Engineering, University of Debrecen, \\ 4028 Debrecen, Ótemető u. 2-4., Hungary \\ * Corresponding author, e-mail: deak.krisztian@eng.unideb.hu
}

Received: 24 August 2018, Accepted: 02 January 2019, Published online: 06 March 2019

\begin{abstract}
This paper considers a comparison of six wavelets for bearing fault diagnosis. Five wavelets Symlet_05, Symlet_08, Daubechies_04, Daubechies_06, Daubechies_08 are typical ones which are used for fault diagnosis due to several researches. The purpose is to design a new discrete wavelet which has higher efficiency to reveal minor defects on the bearing rings. Defects derive from either manufacturing or operational problems. Detecting of tiny manufacturing defects, especially manufacturing grinding marks is quite difficult due to their special geometrical shapes, however they can cause serious problems in machines during operation. Therefore, it is an important task to diagnose these marks with the most adequate methods. The transient vibration signal model of the defect is established for signals generated by tapered roller bearing on the outer race. The wavelet creation used the sub-optimal algorithm devised by Chapa and Rao. The applicability of the matched wavelet is tested for identifying this kind of bearing failure. The new wavelet analysis and synthesis filter coefficients are determined which define the designed wavelet. To determine the efficiency of the designed wavelet and to establish comparison with the other wavelets, a test-rig was constructed with high-precision measuring sensors and devices. By using the Maximum Energy-to-Shannon Entropy criteria the efficiency of the wavelets is determined. The designed wavelet is found to be the most effective to detect the manufacturing fault compared to the others in this article. The final purpose is not only to detect the faults but to determine their sizes. By analyzing the entry points of the rollers into the defects, the de-stressing point and the exit points of the rollers from the defects the width of the grinding marks is calculated. It is proved that the new-designed wavelet obtains the most precise way for fault width measurement. Finally, the size of the failure is measured by a contact type Mahr Perthometer to compare the results to the calculated parameters and validate them. The width deviation is only $1.18 \%$ in the case of the new-designed wavelet which is remarkable precision level for bearing fault analysis.
\end{abstract}

\section{Keywords}

wavelet analysis, bearing vibration analysis, diagnosis condition monitoring

\section{Introduction}

Bearing faults are researched by many researchers because of its importance in industrial applications. Patel et al. [1] applied envelope method to diagnose defects of deep groove ball bearings. Khanam et al. [2] used Kalman and $\mathrm{H}$ filters to measure bearing faults especially in special circumstances where low signal-to-noise ratio made difficult to identify the useful components of the vibration signal. Al-Ghamd and Mba [3] applied acoustic (AE) method combined with the spectrum vibration analysis to investigate the bearing outer race defect width directly from the raw signal. Elforjani and Mba [4] proved the effectiveness of acoustic emission method in the case of slow-speed bearings. Sawalhi and
Randall [5] made researches to determine the fault size of the bearings from the vibration signal by analyzing the entry and exit signals of the roller into and from the defect.

Peng and Chu [6] because proved the outstanding computational efficiency of the wavelets for fault feature extraction, singularity detection for signals, denoising and extraction of the weak signals. Prabhakar et al. [7] applied discrete wavelet transform with Daubechies-4 (db04) mother wavelets to analyze the combination of different faults on the races of ball bearings. Combination of envelope spectrum and wavelet transform for extraction of defect problems in bearings were used by Shi et al. [8]. 
Nikolaou and Antoniadis [9] applied complex shifted Morlet wavelets to analyzing vibration signals generated by rolling element bearings.

Symlet wavelets were used efficiently in the study of Kumar and Singh [10]. In their study tapered roller bearings were analyzed to determine the fault size on the outer ring.

Tóth and Tóth [11, 12] revealed artificial faults of the inner rings of deep groove bearings by wavelet analysis. A realistic signal model of ball bearings with inner race fault was created to design a new wavelet to reveal the defect more efficiently from the vibration signature.

Junsheng et al. [13] used an impulse response wavelet to analyze faults in a roller bearing with CWT. Their wavelet is simply an exponentially damped sinusoid. Sheen [14] effectively applied the Morlet wavelet in the envelope detection for the vibration signal and found it also useful in the defect diagnosis of bearing vibrations. The application of the complex Morlet wavelet with SVM classifier is suggested in [15] for fault diagnosis of ball bearings having localized defects on various bearing components. Liu et al. [16] suggested an automatic feature extraction algorithm for bearing fault diagnosis using a correlation filter-based matching pursuit.

Tewfik et al. [17] worked out a design method that matches a wavelet to the time domain form of a signal. Chapa and Rao [18] developed an algorithm that searches for a matching wavelet in frequency domain. Their method is capable of designing Meyer wavelets that approximate the wavelet amplitude and phase spectra separately. The cost function is the minima of the Mean Squared Error (MSE), calculated from the amplitude spectra and group delay of the signal and the wavelet.

Applicability of SVM is proved in different fields of engineering system analysis. Mankovits et al. [19] executed the optimization of the shape of axi-symmetric rubber bumpers by support vector regression that shows the efficiency of the method in other engineering applications. Vámosi [20] solved a nonlinear classification problem of rubber elements with support vector classification. Manickam [21] applied soft computing methods, back propagation neural network for prediction of shell moulding parameters that showed the efficiency of machine learning methods. Kalácska et al. [22] analyzed the sliding properties of steels on other materials in their research combined with classification. Deák et al. [23] investigated the defect size of tapered roller bearings with wavelet transform by entropy optimization.
Mankovits et al. [24] analyzed the behavior of rubber parts under mechanical stress and applied machine learning techniques such as SVM for optimization.

Wéber and Hős [25] investigated experimental and numerical analysis of hydraulic transients in the presence of air valve.

Mallat [26] created algorithm to analyze bearing fault signal and make the correlation signal to the original parameters to reveal the defects more efficiently.

\section{Methods}

\subsection{Wavelets, Continuous Wavelet Transform and Scalogram}

Wavelet transform is continuous or discrete and it is calculated by the convolution of the signal and a wavelet function. A wavelet function is a small oscillatory wave, which contains both the analysis and the window function. Continuous wavelet transform (CWT) generates the two dimensional maps of coefficients that is called scalogram.

$$
C W T_{f}(a, b)=\frac{1}{\sqrt{a}} \int_{-\infty}^{\infty} f(t) \cdot \Psi^{*}\left(\frac{t-b}{a}\right) d t,
$$

where $a$ is the scale parameter, $b$ is the translation parameter, $f(t)$ is the signal in time domain, $\Psi$ is the "mother" wavelet, and $\Psi^{*}$ is the complex conjugate of $\Psi$ [9].

Discrete wavelet transform (DWT) applies filter banks for the analysis and synthesis of a signal. Filter banks contain wavelet filters and extract the frequency content of the signal in the pre-determined subbands. The discrete wavelet transform is derived from the discretization of continuous wavelet transform by adopting the dyadic scale and translation to reduce the computational time and can be expressed by Eq. (2):

$$
D W T_{s}(j, k)=\frac{1}{\sqrt{2^{j}}} \int_{-\infty}^{\infty} s(t) \cdot \Psi^{*}\left(\frac{t-2^{j} k}{2^{j}}\right) d t,
$$

where $j$ and $k$ are integers, $2^{j}$ and $2^{j} k$ represent the scale and translation parameter respectively. The original signal $s(t)$ passes through a set of low pass and high pass filters emerging as low frequency (approximations, $a_{i}$ ) and high frequency (details, $d_{i}$ ) signals at each decomposition level $i$. They are usually finite impulse response filters whose impulse response (or response to any finite length input) is of finite duration, because it settles to zero in finite time. Therefore, the original signal $s(t)$ can be written as [10]:

$$
s(t)=a_{n}+\sum_{i=l}^{n} d_{i} .
$$


The wavelet function $\Psi$ and scaling function $\varphi$ can be defined as:

$$
\begin{gathered}
\Psi_{j, k}[t]=2^{\frac{j}{2}} \sum_{k} d_{j, k} \Psi\left[2^{j} t-k\right] \\
\varphi_{j, k}[t]=2^{\frac{j}{2}} \sum_{k} c_{j, k} \varphi\left[2^{j} t-k\right]
\end{gathered}
$$

where $d_{j, k}$ and $c_{j, k}$ are the wavelet and scaling coefficients at scale $j[28]$.

Assuming the signal $X[t]=\left(v_{0}, \ldots, v_{N-1}\right)$, the sampling number is $N=2^{j}$, where $j$ is an integer. For $X_{j}[t]$ at scale $j$ decomposed to scale $j-1$ of $D W T$ model can be defined as [30]:

$$
\begin{aligned}
& D W T\left(X_{j}[t]\right) \\
= & 2^{\frac{j-1}{2}} \cdot\left(\sum_{k=0} c A_{j-l, k} \varphi\left[2^{j-1} t-k\right]+\sum_{k=0} c D_{j-l, k} \Psi\left[2^{j-1} t-k\right]\right) \\
0 \leq & k \leq \frac{N}{2^{j}}-1
\end{aligned}
$$

where

$$
\begin{aligned}
c A_{j-l, k}=\sum_{h=0}^{\frac{N}{2^{j}}-1} c_{j, h} v_{j, h+2 n}, & c D_{j-l, k} & =\sum_{h=0}^{\frac{N}{2^{j}}-1} d_{j, h} v_{j, h+2 n} \\
d_{h}=(-1)^{h} c_{2 p-l-h}, & p & =\frac{N}{2^{j}} .
\end{aligned}
$$

The scalogram, defined as the squared magnitude of $C W T$, always has non-negative, real-valued time-frequency (scale) distribution. This transformation conserves energy. Its resolution in the time-frequency plane depends on the scale parameter.

$$
\begin{aligned}
S C\{f(a, b)\} & =|\operatorname{CTW}\{f(a, b)\}|^{2} \\
& =\left|\int_{-\infty}^{+\infty} f(t) \frac{1}{\sqrt{a}} \psi\left(\frac{t-b}{a}\right) d t\right|^{2} .
\end{aligned}
$$

Polynomial spline functions such as Fourier transform, frequency response function, satisfies orthogonality conditions [26]. Shannon, Meyer, Battle-Lemarie and Daubechies wavelet are widely applied which have compactly support.

Meyer wavelet is defined through the scaling function as $[12,18]$

$$
\hat{\phi}(\omega)=\left\{\begin{array}{cc}
1, & |\omega|<\frac{2 \pi}{3} \\
\cos \left[\frac{\pi}{2} v\left(\frac{3}{2 \pi}|\omega|-1\right)\right], & \frac{2 \pi}{3} \leq|\omega| \leq \frac{4 \pi}{3} \\
0, & \text { otherwise }
\end{array}\right.
$$

where $v(\omega)$ is a tapering function.

\subsection{Matching Wavelet to a Given Signal}

Chapa and Rao [18] searched a technique which is an efficient method for designing Meyer wavelets to match band-limited signals. Their method directly matches a wavelet to the signal. It requires some pre-determined conditions on the wavelet spectrum amplitude and phase. The band-limited scaling function and the bandlimited are determined which are sufficient conditions for an Orthonormal Multiresolution Analysis (OMRA). They also derive constraints on the structure of the wavelet phase. The matching algorithm is sub-optimal which means it matches to the wavelet amplitude and phase independently [12].

\subsection{Matching Wavelet Amplitude}

Discrete form of the refinement equation should be used that can be expressed from a wavelet [18]:

$\left|\hat{\phi}\left(\frac{\pi k}{2^{l}}\right)\right|^{2}=\sum_{p=0}^{l}\left|\hat{\psi}\left(\frac{2 \pi k}{2^{p}}\right)\right|^{2}$.

Chapa and Rao [18] determined the wavelet spectra $\hat{\psi}(\omega)$ to prove the orthonormality and an error function:

$E(Y, a)=\int_{\frac{2 \pi}{3}}^{\frac{8 \pi}{3}}[W(\omega)-a Y(\omega)]^{2} d \omega$

where

$W(\omega)=\left|F(\omega)^{2}\right|$

$Y(\omega)=|\hat{\psi}(\omega)|^{2}$

$a$ : scaling coefficient.

The algorithm searches for the extreme value of cost function in a discrete form. Symmetric property of the wavelet function is described by Eq. (12)

$\sum_{p=0}^{l} \sum_{m=-\infty}^{+\infty} Y\left(\frac{2^{l}}{2^{p}}\left(k+2^{l+1} m\right)\right)=1$

where

$\frac{2^{l-1}}{3}<\left|\frac{2^{l}}{2^{p}}\left(k+2^{l+1} m\right)\right|<\frac{2^{l+2}}{3}$.

This condition generates a set of $L$ linear equality constraints in $Y(k)$ of the form

$\sum_{i=1}^{L} \alpha_{i k} Y(k)=1$ for $k=\frac{2^{l}}{3}, \ldots, \frac{2^{l+2}}{3}$,

where $\alpha_{i k} \in\{0,1,2\}$. This equation can be expressed in matrix form as $\boldsymbol{A} \boldsymbol{Y}=1$, where

$\boldsymbol{A}=\left\{\alpha_{i j} \in\{0,1,2\} ; i=1, \ldots, L ; j=1, \ldots, 2^{l}\right\}$. 
The error function can be given in Eq. (13):

$E=\frac{(\boldsymbol{W}-a \boldsymbol{Y})^{T}(\boldsymbol{W}-a \boldsymbol{Y})}{\boldsymbol{W}^{T} \boldsymbol{W}}$.

Matching amplitudes can be expressed by Eq. (14):

$Y=\frac{1}{a} \boldsymbol{W}+\boldsymbol{A}^{T}\left(\boldsymbol{A} \boldsymbol{A}^{T}\right)^{-1}\left(1-\frac{1}{a} \boldsymbol{A} \boldsymbol{W}\right)$

where

$a=\frac{1^{T}\left(\boldsymbol{A} \boldsymbol{A}^{T}\right)^{-1} \boldsymbol{A} \boldsymbol{W}}{1^{T}\left(\boldsymbol{A} \boldsymbol{A}^{T}\right)^{-1} 1}$.

\subsection{Matching Wavelet Phase}

Mean-square error (MSE) criteria is applied for phase matching which is similar to amplitude matching, however it uses group delay of the signal.

The group delay of a signal is defined as the first order, negative derivative of the phase

$\tau(\omega)=-\frac{d \theta(\omega)}{d \omega}$.

Specific constraints on the structure of the wavelet phase, can be expressed in terms of the phase of the scaling function

$\theta_{\psi}(\omega)=-\frac{\omega}{2}-\theta_{\phi}(\omega+2 \pi)+\theta_{\phi}\left(\frac{\omega}{2}+\pi\right)+\theta_{\phi}\left(\frac{\omega}{2}\right)$

where $\theta_{\phi}$ is the phase of scaling function $\phi(\omega)$ and $\theta_{\psi}$ is the phase of wavelet $\psi(\omega)$.

The wavelet phase is a symmetric, $2 \pi$ periodic function. The method models one period of the negative of the group delay, denoted by $\lambda_{T}(\omega)$ as a polynomial of order $R$ [18]:

$\lambda_{T}(\omega)=\sum_{r=0}^{\frac{R}{2}} c_{r} \omega^{2 r} \Pi\left(\frac{\omega}{2}\right)$

where

$\lambda(\omega)=-\tau(\omega)$

$\Pi(\omega)\left\{\begin{array}{lc}1, & -\frac{1}{2} \leq \omega<\frac{1}{2} \\ 0, & \text { otherwise }\end{array}\right.$

$c_{r}=$ polynominal coefficients.

By replicating one period of the group delay at every $2 \pi$ interval, the group delay of the wavelet is modelled as the $2 \pi$ periodic polynomial of order $R$ [11] in discrete it can be written as

$$
\lambda(n)=\sum_{r=0}^{R / 2} c_{r} \sum_{k=-P / 2}^{P / 2-1}(n-k T)^{2 r} \Pi\left(\frac{n-k T}{T}\right),
$$

where

$$
\Delta \omega=\frac{2 \pi}{T}, \quad P=\frac{N}{T},
$$

$N$ is the number of samples, $-\frac{N}{2} \leq n<\frac{N}{2}$.
With vector notation, the group delay is expressed as

$\lambda=\boldsymbol{B} c$,

where

$\boldsymbol{b}_{n, r}=\sum_{k=\frac{P}{2}}^{\frac{P}{2}}(n-k T)^{2 r} \Pi\left(\frac{n-k T}{T}\right)$.

Negatives of the group delays $\Lambda_{\Psi}$ and $\Lambda_{\phi}$ can be expressed in terms of $\lambda(\omega)$.

Applying $\Gamma_{\Psi}(\omega)=\Lambda_{\Psi}+\frac{1}{2}$ substitution, we get:

$\Gamma_{\Psi}=\boldsymbol{D}_{\Psi} c=-\frac{1}{2} \boldsymbol{B}_{\left(\frac{q+T}{2}\right)}+\sum_{m=2}^{\infty} 2^{-m} \boldsymbol{B}_{\frac{q}{2^{m}}}$

$\Gamma_{\phi}=\boldsymbol{D}_{\phi} c=\sum_{m=1}^{\infty} 2^{-m} \boldsymbol{B}_{\frac{q}{2^{m}}}$.

The matching process minimizes the weighted error between the group delay of the wavelet $\Gamma_{\Psi}$ and the desired signal $\Gamma_{F}[11,18]$. The approximation is performed only in the pass-band, thus a weighting function $\Omega(n)$ is calculated from the result of the amplitude matching process

$\gamma=\sum_{n=-N / 2}^{N / 2-1}\left(\Omega(n)\left(\Gamma_{F}(n)-\Gamma_{\Psi}(n)\right)\right)^{2}$,

where

$\Omega(n)=\frac{Y(n)}{\sum Y(n)}$.

The optimal values of the polynominal coefficients can be obtained by solving Eq. (23):

$\nabla_{c} \gamma=0$

\subsection{Matching a Wavelet to Signal Model of Bearing Vibration}

The manufacturing fault on the outer ring causes an amplitude modulation that is more emphasized in the load zone. One of these impulses can be described as

$x(t)=A \cdot t^{n} \cdot e^{-C \cdot t} \cdot \sin \left(\omega_{n} \cdot t\right), \quad \omega_{n}=2 \pi f_{n}$

where $f_{n}$ is the $n^{\text {th }}$ natural frequency, $C$ is a damping factor, $A$ is the initial amplitude and $n$ is an exponential behavior the transient.

In order to create a new wavelet basis function, we used 1024 samples of time domain data of a transient vibration signal described with $A=28.74, n=2.748, C=17.34$, and $\omega_{0}=265.28$ equation parameters. In order to get accurate parameters, the transient signal was analyzed as Figs. 1-2 show. 


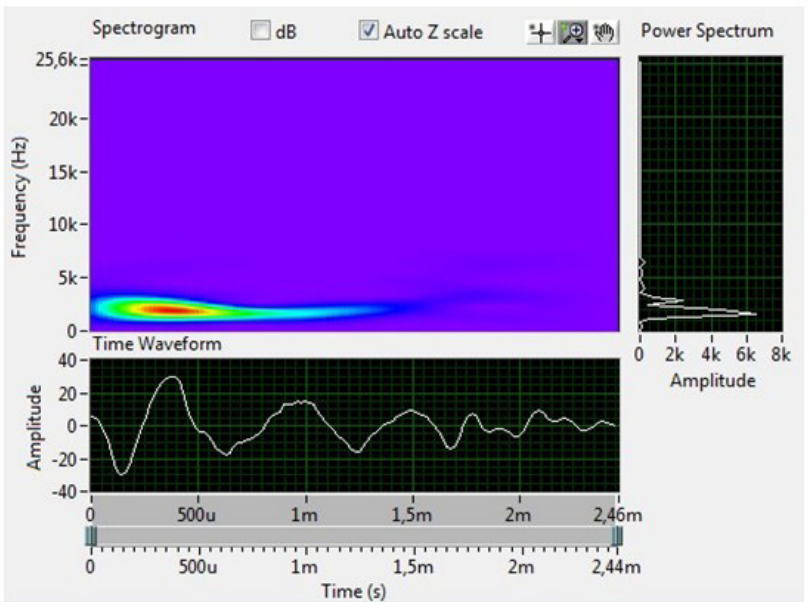

Fig. 1 Transient signal of the outer-ring grinding fault with its spectrogram

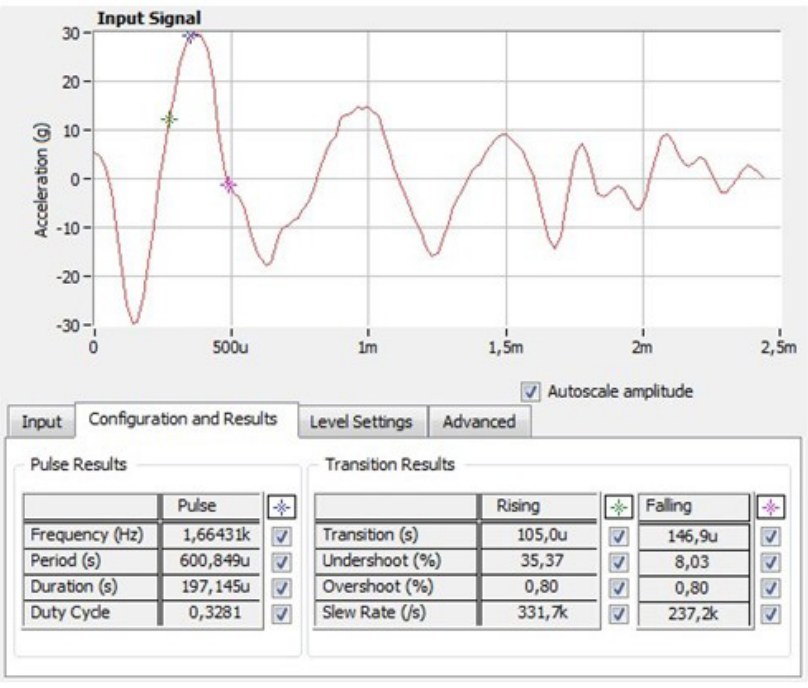

Fig. 2 Parameters of the measured transient signal for model creation

Using the parameters it provides close correlation to the envelope spectrum of the transient with minimal meansquare error which is a sufficient optimization of the cost function as it is perceived in Figs. 3-4.

\subsection{New wavelet design parameters}

We applied Chapa and Rao's amplitude and phase matching algorithm [18] on the transient signal model of the bearing vibration. The new wavelet amplitude spectra match the amplitude spectra of the transient very well in the passband. The MSE of the matching is 0.213 . For phase matching we used a non-linear polynomial.

Since the new wavelet basis cannot be given in closed form we give the filter coefficients in Table 1. It is clear from the table that Quadrature Mirror Filters (QMF) have no compact support.

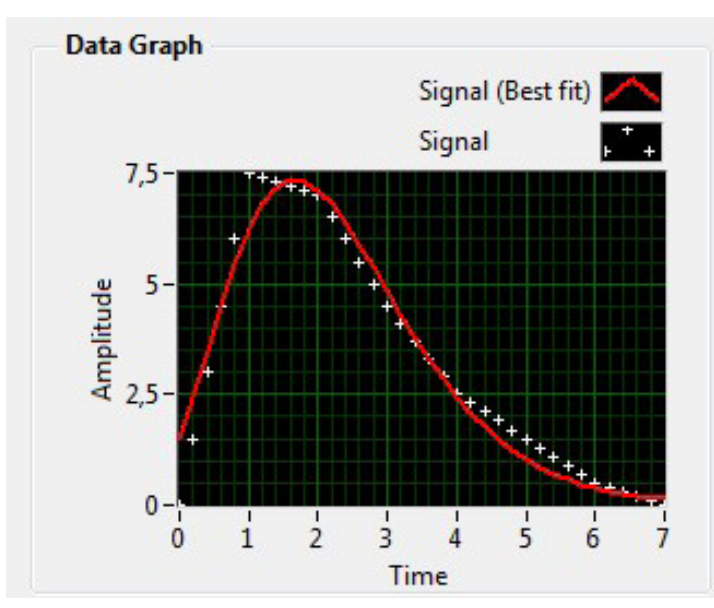

Fig. 3 Envelope model of the transient signal

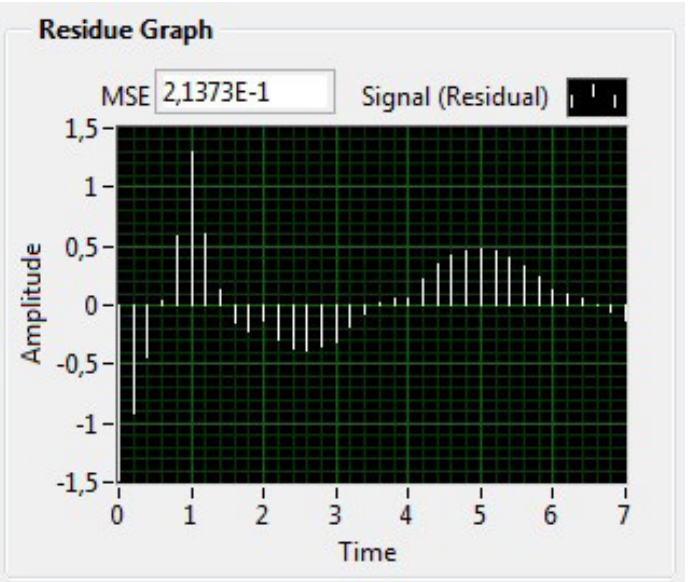

Fig. 4 Mean Square Error of the transient from the envelope values

Table 1 New-designed wavelet filter coefficients

\begin{tabular}{|c|c|c|c|c|}
\hline \multirow[b]{2}{*}{ No. } & \multicolumn{2}{|c|}{ Synthesis filters } & \multicolumn{2}{|c|}{ Analysis filters } \\
\hline & $\begin{array}{c}\text { Lowpass } \\
(H 0)\end{array}$ & $\begin{array}{c}\text { Highpass } \\
\quad(H 1)\end{array}$ & $\begin{array}{c}\text { Lowpass } \\
\quad(G 0)\end{array}$ & $\begin{array}{c}\text { Highpass } \\
(G 1)\end{array}$ \\
\hline 0 & -0.07 & 0.0032 & 0.00 & -0.0657 \\
\hline 1 & -0.14 & -0.0069 & -0.01 & 0.142 \\
\hline 2 & 0.07 & 0.0025 & 0.00 & 0.0698 \\
\hline 3 & 0.57 & 0.0258 & 0.03 & -0.5680 \\
\hline 4 & 0.73 & 0.0023 & 0.00 & 0.7290 \\
\hline 5 & 0.28 & -0.0529 & -0.05 & -0.2811 \\
\hline 6 & -0.12 & -0.0375 & 0.04 & -0.1241 \\
\hline 7 & -0.03 & 0.0129 & 0.13 & 0.0328 \\
\hline 8 & 0.13 & 0.0330 & -0.03 & 0.1300 \\
\hline 9 & 0.04 & -0.1230 & -0.12 & -0.0373 \\
\hline 10 & -0.05 & -0.2810 & 0.28 & -0.0531 \\
\hline 11 & 0.00 & 0.7280 & 0.73 & 0.0022 \\
\hline 12 & 0.03 & -0.5670 & 0.57 & 0.0258 \\
\hline 13 & 0.00 & 0.0704 & 0.07 & 0.0026 \\
\hline 14 & -0.01 & 0.1420 & -0.14 & -0.0068 \\
\hline 15 & 0.00 & -0.0658 & -0.07 & -0.0032 \\
\hline
\end{tabular}


Analysis and synthesis functions of the new-designed wavelet and the coefficients of analysis and synthesis filters are in Fig. 3. By limiting the filter coefficients to this range, we cannot reconstruct the original data from the wavelet coefficients completely. The fewer coefficients we use, the more error we get during reconstruction.

Zeros of $G 0$ and $H 0$ of the new-designed wavelet is shown of the Figs. 5-6.

\section{Experimental test}

The purpose of this paper is to apply the new-designed wavelet for detection and fault size determination of bearings with real manufacturing fault. For this purpose, test-rig (Fig. 8) had been constructed that provides accurate measurements.

The shaft in the test rig is supported by two tapered roller bearings. The one under investigation is No. 30205 tapered roller bearing. Defect on the outer race is a line (rectangular) shape grinding defect (Fig. 15) which is under analysis here. The shaft is driven by an alternating current motor of $0.75 \mathrm{~kW}$ (made by Cemer), frequency of $50 \mathrm{~Hz}$, and nominal speed of $2770 \mathrm{rpm}$ which is reduced to $1800 \mathrm{rpm}$ with variable speed drive device. Rubber V-belt between the electric engine and the shaft provides smooth running and low vibration which help accurate and precise measurements. Rubber bumpers are installed to reduce vibration of the electric motor to the bearing housing in order to minimize harmful vibrations. The arrangement
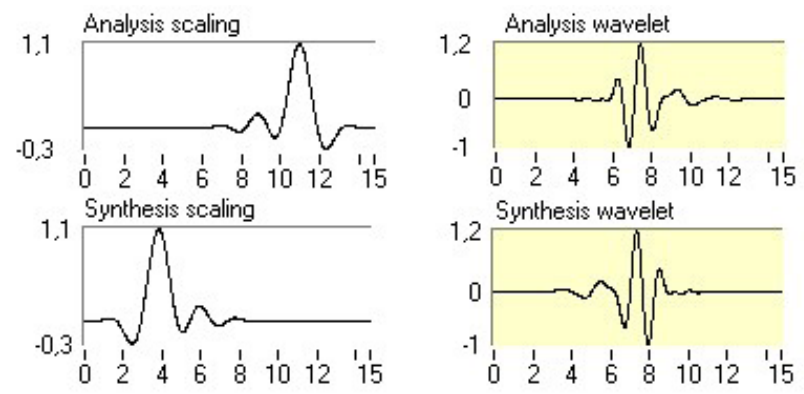

Fig. 5 Analysis and synthesis functions of the new-designed wavelet
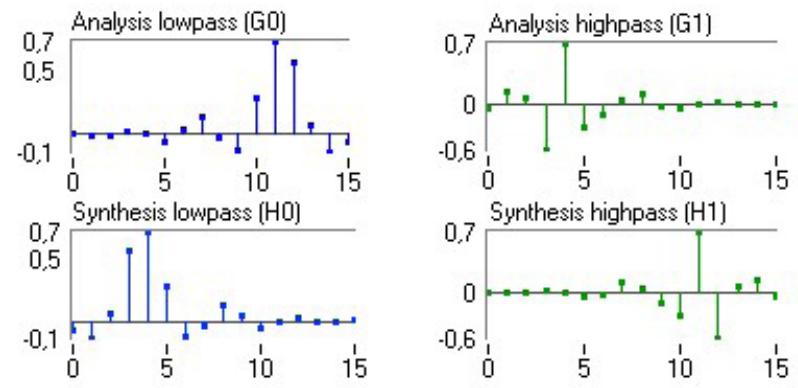

Fig. 6 Values of the analysis and synthesis filters of the new-designed wavelet

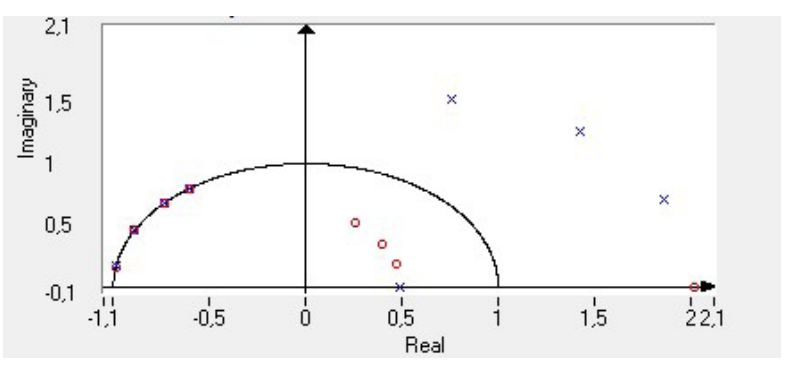

Fig. 7 Zeros of $G 0$ and $H 0$ of the new-designed wavelet
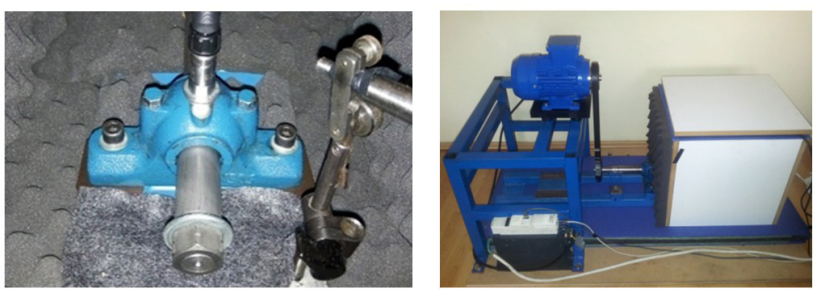

Fig. 8 Bearing test-rig with high-precision measurement devices

provides option of different speeds controlled by Schneider ATV32HU22M2 variable speed drive device. In the experiment the speed of the shaft is measured using an optical tachometer with digital display to check the speed fluctuations. Test bearing is spanned by screw mechanism to supply the sufficient axial force to the measurements. Constant spanning force during the measurements is measured by strain gauges in Wheatstone Bride mode on the basis of difference in voltage measurement.

NI 9234 dynamic signal acquisition is used in the experiments with 4-channels to vibration measurements from integrated electronic piezoelectric (IEPE) and non-IEPE sensors. The NI 9234 delivers $102 \mathrm{~dB}$ of dynamic range. Input channels simultaneously digitize signals at rates up to $51.2 \mathrm{kHz}$ per channel with built-in anti-aliasing filters. PCB IMI 603C01 vibration transducer is used which is an industrial type platinum stock piezoelectric sensor with low noise level, sensitivity of $100 \mathrm{mV} / \mathrm{g}$ and frequency range of 0.27 to $10 \mathrm{kHz}$ with top exit 2-pin connector. The accelerometer is placed on the previously ground surface of the top of the bearing house with screw mechanism perpendicular to the axis of the rotation of the shaft. 32 bit AMD Athlon II X2 M300 $2.0 \mathrm{GHz}$ processor is used for data processing. For visual validation of the defect sizes on the bearing rings Garant MM1-200 video microscope is applied that is an incremental measuring system, built-in image processing with 1.3 megapixel color camera. Furthermore, Mahr MMQ 200 with precision roundness measuring axis, motorized vertical and horizontal measuring axis is used for roundness deviation measurement to determine both width and depth of the grinding marks. 
Time domain of the recorded signal provide a noisy signal, however it has much higher SNR (signal-to-noise ratio) than a signal without using the sophisticated bearing test-rig.

Frequency domain (Fig. 9) shows a $2.09 \mathrm{kHz}$ peak which is the frequency of the bearing components in this experience it is called structural frequency as well.

Since the signal of transient pulses generated by the grinding mark on the outer raceway of a tapered roller bearing was very similar to the typical analysis wavelets, we can conclude that wavelets are a reasonable choice that is sure to yield good results. However, several experiments proved that designed wavelets for the special faults provide much better result for fault detection and size determination than the typical Symlet, Daubechies wavelets which has not so specialized parameters for the actual fault manner on the bearing.

\section{Matching wavelets and ranking by Energy-Entropy method}

The main purpose of the experiment is to compare the measured transient signal to the signals generated by the wavelets. Figs. 10-12 show the Daubechies_08 gives the worst correlation to the measured signal therefore it is assumed to be not effective for detecting the bearing fault. By visual inspection it is clear that the new-designed wavelet provide a very good correlation to the measured signal. However, visual comparison is not a very precise method to determine the efficiency of a wavelet. For this purpose we used a method that provided to be a reliable method for wavelet efficiency analysis called Maximum Energy-to-Shannon Entropy criteria.

The combination of the energy and Shannon entropy content of the wavelet coefficients of the signal, denoted by Energy to Shannon Entropy ratio is an appropriate

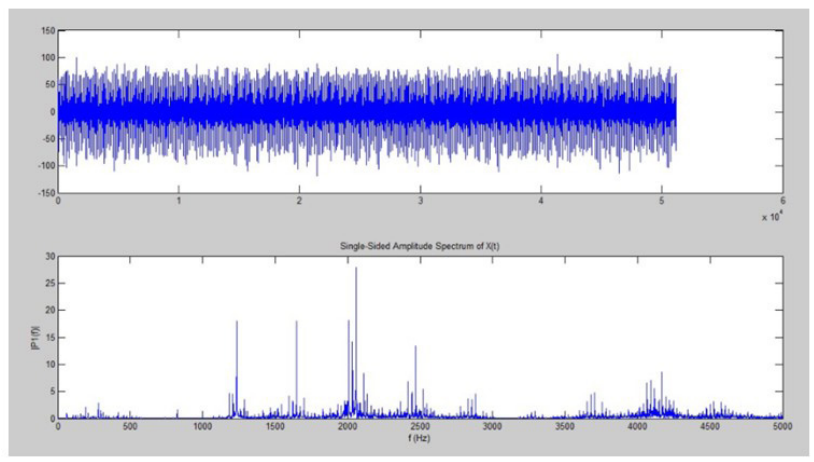

Fig. 9 Time domain (above) and frequency domain (below) of the measurement
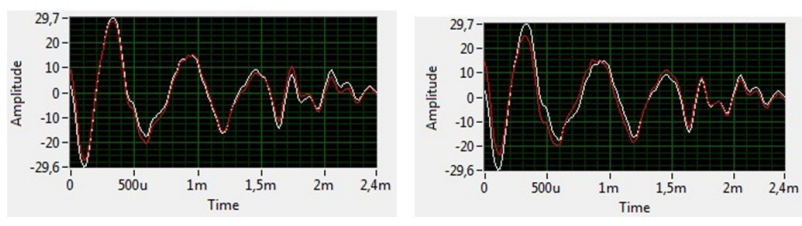

Fig. 10 Symlet_08 wavelet (left) and db_06 (right) matching with the original signal
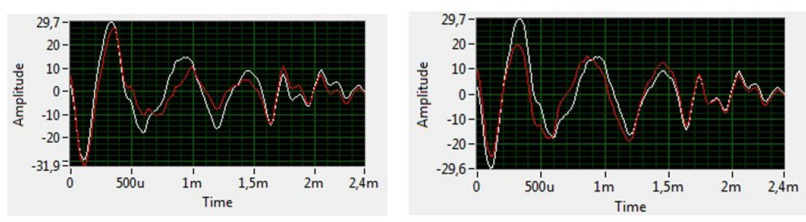

Fig. 11 db 04 wavelet (left) and Symlet 05 (right) matching with the original signal
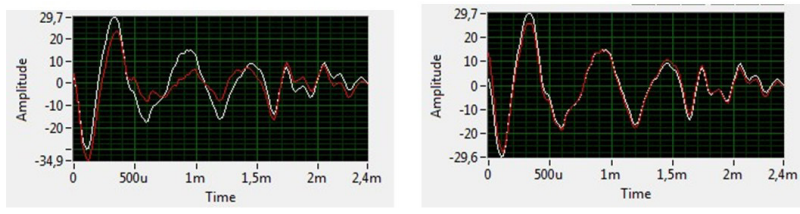

Fig. 12 db_08 wavelet (left) and new-designed (right) matching with the original signal

indicator to choose the best wavelet for diagnosis and it can be calculated in Eq. (24) [15]

$\xi(n)=E(n) / S_{\text {entropy }}(n)$.

The energy content of signal wavelet coefficients is given as

$E(n)=\sum_{i}^{m}\left|C_{n, i}\right|^{2}$,

where $m$ is the number of the wavelet coefficients, $C_{n, i}$ is the $i^{\text {th }}$ wavelet coefficient of $n^{\text {th }}$ scale.

The entropy of signal wavelet coefficients is given by

$S(n)=-\sum_{i=1}^{m} p_{i} \log _{2} p_{i}$,

where $\left(p_{1}, \ldots, p_{n}\right)$ is the energy distribution of the wavelet coefficients defined by

$p_{i}=\left|C_{n, i}\right|^{2} / E(n)$.

In this experiment, three measurements were established as Table 2 shows $E / S$ values were determined by Labview VI for this purpose from the measured signal.

Values, after calculating the mean values of $E / S$ ratio, are presented in Fig. 13. It is observed that new-designed wavelet gives the highest value that indicates to be the most efficient wavelet for both fault detection and fault size estimation. 
Table $2 E / S$ values of the wavelets by the number of measurements

\begin{tabular}{lcccc}
\hline$E / S$ values & 1st & 2nd & 3rd & Mean \\
\hline Sym5 & 99.65 & 101.12 & 100.67 & 100.48 \\
Sym8 & 121.85 & 122.81 & 123.05 & 122.57 \\
db04 & 88.18 & 87.21 & 86.84 & 87.41 \\
db06 & 117.01 & 115.94 & 116.07 & 116.34 \\
db08 & 84.28 & 82.86 & 84.50 & 83.88 \\
New_Designed & 155.12 & 154.18 & 155.23 & 154.84 \\
\hline
\end{tabular}

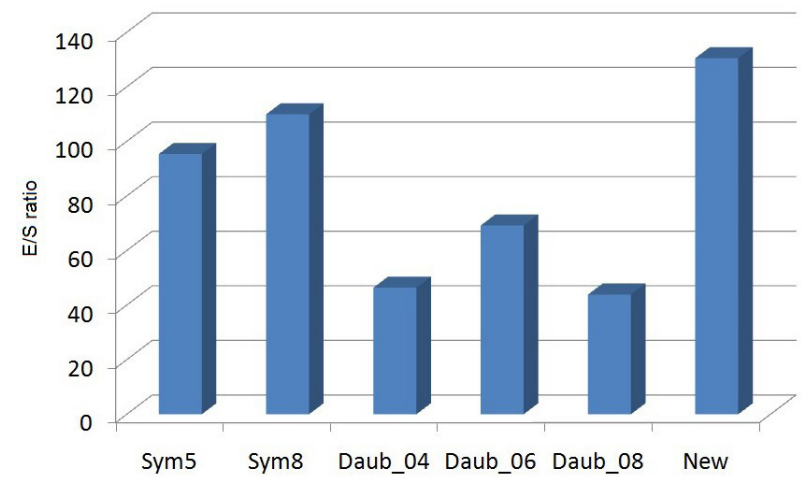

Fig. 13 Rank of the $E / S$ values of the wavelets

Fault detection procedures based on time-frequency methods usually rely on visual observation of contour plots. It is also known that if the wavelet matches well with the shape of the signal at a specific scale and location a large transform value is obtained. However, a low transform value is obtained if the signal and wavelet do not correlate well. To avoid defects of visual observation a more precise way of determining the best suited wavelet is presented here by $E / S$ parameters.

The scalogram (Fig. 14) clearly shows the time-frequency location of transients. These pulses do not appear at each rotation. They seem to be random signals that might come from the test rig. In contrast, periodically repeating transient pulses are clearly seen on the scalogram of the pitted bearing. Their time-period and frequency can be numerically given. The application of this method reduces the incorrect evaluation of vibration data and can be a valuable supplement to conventional condition monitoring methods.

Comparing the two scalogram representations we notice that the new-designed wavelet provided a more realistic result and gave better energy concentration. The scalogram calculated by the matching wavelet provided better time localization, but its frequency localization is less accurate than that of the designed wavelet.

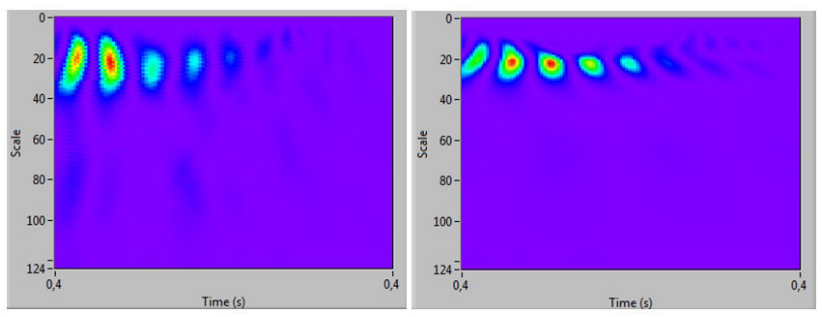

Fig. 14 Comparison of the scalograms of the Daub_08 (left) and the new-designed wavelet (right)

\section{Bearing fault width measurement with optical validation}

To verify the precision of the previous measurements image processing is applied to reveal the exact geometrical size of the ground defect on the outer race. Garant MM1-200 video microscope is used to analyze and measure the grinding marks on the rings and Mahr MMQ 200 contact equipment (Fig. 15) was applied for roundness deviation measurement of the outer rings to determine the depth of the grinding marks on the rings.

Analysis of the entry point, the exit point and the de-stressing point of the roller for the purpose of fault size calculation [10]. At point $A$, the roller strikes the groove base with high impact which results in re-stressing and high impulse in signal. After this event the roller remains in contact with the groove base for some time and during this period impulses due to the rough surface of groove are observed as Fig. 16 presents.

When the roller comes in contact with the point $B$ it again generates high amplitude in the signal and beyond this (i.e. after $B$ ) progressive decrease in amplitude of signal is observed due to elastic damping of bearing element.

For fault size estimation fault frequencies are calculated which are $B P F O=206.18 \mathrm{~Hz}, B P F I=287.15 \mathrm{~Hz}$, $F T F=12.88 \mathrm{~Hz}, B S F=89.96 \mathrm{~Hz}$ in this experiment at $1800 \mathrm{rpm}$. Regarding the BPFO frequency analysis was made at $3^{\text {rd }}$ detail level $\left(\mathrm{cD}_{3}\right)$ from 1.25 to $2.5 \mathrm{kHz}$ by Multiresolution analysis (MRA). Fault parameter can be calculated by Eq. (28) [10]

$L_{O D}=\pi \cdot \Delta t \cdot D_{O I} \cdot F T F=1713.74 \cdot \Delta t$.

Finally, the measurement of defect width had been measured by the different wavelets. As Table 3 shows, the new-designed wavelet provides the most accurate geometrical fault determination. This result is in correlation to Figs. 7-9. Figs. 7-9 show the same result graphically. 


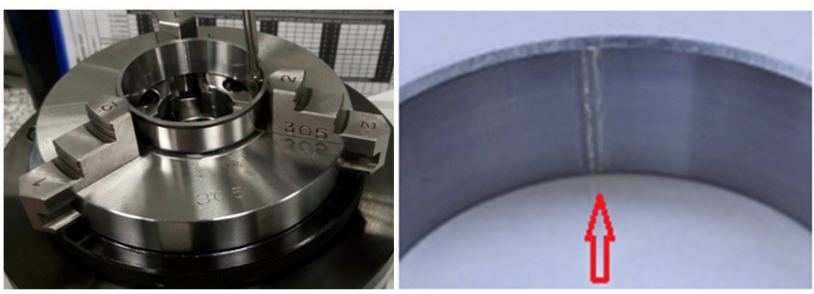

Fig. 15 Measurement of the outer ring of the tapered roller bearing with grinding defect of $1.2492 \mathrm{~mm}$ (left) and its enlarged image (right)

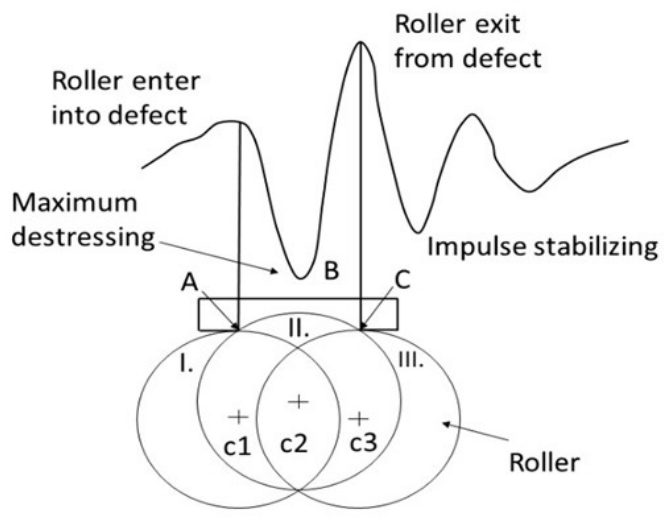

Fig. 16 Analysis of the transient impulse presenting the entry and exit points of the roller into the grinding defects on the outer ring

\section{Conclusion}

This paper showed the creation of a new wavelet that matches the transient vibration response generated by a

\section{References}

[1] Patel, V. N., Tandon, N., Pandey, R. K. "Defect Detection in Deep Groove Ball Bearing in Presence of External Vibration Using Envelope Analysis and Duffing Oscillator", Measurement, 45(5), pp. 960-970, 2012.

https://doi.org/10.1016/j.measurement.2012.01.047

[2] Khanam, S., Tandon, N., Dutt, J. K. "Fault Identification of Rolling Element Bearings from Vibration Signals: An Application of Kalman and $\mathrm{H}_{\infty}$ Filters", In: $10^{\text {th }}$ International Conference on Vibrations in Rotating Machinery (VIRM10), London, UK, 2012, pp. 703-713.

https://doi.org/10.1533/9780857094537.11.703

[3] Al-Ghamd, A. M., Mba, D. "A Comparative Experimental Study on the Use of Acoustic Emission and Vibration Analysis for Bearing Defect Identification and Estimation of Defect Size", Mechanical Systems and Signal Processing, 20(7), pp. 1537-1571, 2006. https://doi.org/10.1016/j.ymssp.2004.10.013

[4] Elforjani, M., Mba, D. "Accelerated Natural Fault Diagnosis in Slow Speed Bearings with Acoustic Emission", Engineering Fracture Mechanics, 77(1), pp. 112-127, 2010. https://doi.org/10.1016/j.engfracmech.2009.09.016

[5] Sawalhi, N., Randall, R. B. "Vibration Response of Spalled Rolling Element Bearings: Observations, Simulations and Signal Processing Techniques to Track the Spall Size", Mechanical Systems and Signal Processing, 25(3), pp. 846-870, 2011. https:/doi.org/10.1016/j.ymssp.2010.09.009
Table 3 Comparision of the calculated and measured fault widths of the faults

\begin{tabular}{lcc}
\hline Wavelet & $\begin{array}{c}\text { Calculated } \\
\text { width (mm) }\end{array}$ & $\begin{array}{c}\text { Width } \\
\text { deviation (\%) }\end{array}$ \\
\hline Sym_05 & 1.2875 & 3.07 \\
Sym_08 & 1.2713 & 1.77 \\
Daub_04 & 1.2930 & 3.51 \\
Daub_06 & 1.2765 & 2.19 \\
Daub_08 & 1.2975 & 3.87 \\
New_designed & 1.2639 & 1.18 \\
\hline
\end{tabular}

grinding fault on the outer race of a tapered roller bearing. The transient of the fault was modelized by a mathematical polynomial function to get the signal model. Wavelet creation is based on Chapa and Rao's method [18], where the Meyer wavelet amplitude and phase spectra are matched independently to the signal. It was shown that the new wavelet can be used for detecting transient pulses generated in a bearing. We compared the results with those calculated using the new wavelet since many application reported its successful application. We found the new wavelet had higher efficiency to reveal minor faults on the bearings. We compared five typical wavelets to the new-designed wavelet. Optical validation reinforced our theory that higher Energy-to-Entropy values of the wavelets obtain more precise determination of the geometrical defects.

[6] Peng, Z. K., Chu, F. L. "Application of the Wavelet Transform in Machine Condition Monitoring and Fault Diagnostics: A Review with Bibliography", Mechanical Systems and Signal Processing, 18(2), pp. 199-221, 2004.

https://doi.org/10.1016/S0888-3270(03)00075-X

[7] Prabhakar, S., Mohanty, A. R., Sekhar, A. S. "Application of Discrete Wavelet Transform for Detection of Ball Bearing Race Faults", Tribology International, 35(12), pp. 793-800, 2002. https://doi.org/10.1016/S0301-679X(02)00063-4

[8] Shi, D. F., Wang, W. J., Qu, L. S. "Defect Detection for Bearings Using Envelope Spectra of Wavelet Transform", Journal of Vibration and Acoustics, 126(4), pp. 567-573, 2004. https://doi.org/10.1115/1.1804995

[9] Nikolaou, N. G., Antoniadis, I. A. "Demodulation of Vibration Signals Generated by Defects in Rolling Element Bearings Using Complex Shifted Morlet Wavelets", Mechanical Systems and Signal Processing, 16(4), pp. 677-694, 2002. https://doi.org/10.1006/mssp.2001.1459

[10] Kumar, R., Singh, M. "Outer Race Defect Width Measurement in Taper Roller Bearing using Discrete Wavelet Transform of Vibration Signal", Measurement, 46(1), pp. 537-545, 2013. https://doi.org/10.1016/j.measurement.2012.08.012

[11] Tóth, L., Tóth, T. "Construction of a Realistic Signal Model of Transients for a Ball Bearing with Inner Race Fault", Acta Polytechnica Hungarica, 10(1), pp. 63-80, 2013. https://doi.org/10.12700/APH.10.01.2013.1.4. 
[12] Tóth, L., Tóth T. "On Finding Better Wavelet Basis for Bearing Fault Detection", Acta Polytechnica Hungarica, 10(3), pp. 17-35, 2013. https://doi.org/10.12700/APH.10.03.2013.3.3.

[13] Junsheng, C., Dejie, Y., Yu, Y. "Application of an Impulse Response Wavelet to Fault Diagnosis of Rolling Bearings", Mechanical Systems and Signal Processing, 21(2), pp. 920-929, 2007. https://doi.org/10.1016/j.ymssp.2005.09.014

[14] Sheen, Y.-T. "On the Study of Applying Morlet Wavelet to the Hilbert Transform for the Envelope Detection of Bearing Vibrations", Mechanical Systems and Signal Processing, 23(5), pp. 1518-1527, 2009.

https://doi.org/10.1016/j.ymssp.2009.01.012

[15] Kankar, P. K., Sharma, S. C., Harsha, S. P. "Rolling Element Bearing Fault Diagnosis Using Wavelet Transform", Neurocomputing, 74(10), pp. 1638-1645, 2011.

https://doi.org/10.1016/j.neucom.2011.01.021

[16] Liu, X., Bo, L., He, X., Veidt, M. "Application of Correlation Matching for Automatic Bearing Fault Diagnosis", Journal of Sound and Vibration, 331(26), pp. 5838-5852, 2012. https://doi.org/10.1016/j.jsv.2012.07.022

[17] Tewfik, A. H., Sinha, D., Jorgensen, P. "On the Optimal Choice of a Wavelet for Signal Representation", IEEE Transactions on Information Theory, 38(2), pp. 747-765, 1992. https://oi.org/10.1109/18.119734

[18] Chapa, J. O., Rao, R. M. "Algorithms for Designing Wavelets to Match a Specified Signal", IEEE Transactions on Signal Processing, 48(12), pp. 3395-3406, 2000. https://doi.org/10.1109/78.887001

[19] Mankovits, T., Szabó, T., Kocsis, I., Páczelt, I. "Optimization of the Shape of Axi-Symmetric Rubber Bumpers", Strojniški vestnik Journal of Mechanical Engineering, 60(1), pp. 61-71, 2014. https://doi.org/10.5545/sv-jme.2013.1315
[20] Vámosi, A. "Nemlineáris osztályozásra vezető müszaki probléma megoldása SVC módszerrel" (Nonlinear Classification by SVC Method in Applied Engineering), In: Müszaki Tudomány az Észak-Kelet Magyarországi régióban 2014 Konferencia, Szolnok, Hungary, 2014, pp. 40-47. (in Hungarian)

[21] Manickam, R. "Back Propagation Neural Network for Prediction of Some Shell Moulding Parameters", Periodica Polytechnica Mechanical Engineering, 60(4), pp. 203-208, 2016. https://doi.org/10.3311/PPme.8684

[22] Kalácska, G., Zsidai, L., Keresztes, R., Tóth, A., Mohai, M., Szépvölgyi, J. "Effect of nitrogen plasma immersion ion implantation of polyamide- 6 on its sliding properties against steel surface", Wear, 290-291, pp. 66-73, 2012. https://doi.org/10.1016/j.wear.2012.05.011

[23] Deák, K., Mankovits, T., Kocsis, I. "Optimal Wavelet Selection for Manufacturing Defect Size Estimation of Tapered Roller Bearings with Vibration Measurement using Shannon Entropy Criteria", Strojniški vestnik - Journal of Mechanical Engineering, 63(1), pp. 3-14, 2017. https://doi.org/10.5545/sv-jme.2016.3989

[24] Mankovits, T., Kocsis, I., Portik, T., Szabó, T., Páczelt, I. "Shape Design of Rubber Part Using FEM", International Review of Applied Sciences and Engineering, 4(1), pp. 85-94, 2013. https://doi.org/10.1556/IRASE.4.2013.1.12

[25] Wéber, R., Hős, C. "Experimental and Numerical Analysis of Hydraulic Transients in the Presence of Air Valve", Periodica Polytechnica Mechanical Engineering, 62(1), pp. 1-9, 2018. https://doi.org/10.3311/PPme.10336

[26] Mallat, S. "A Wavelet Tour of Signal Processing", 2nd ed., Academic Press, San Diego, USA, 1999. 